\title{
Frontières
}

\section{La mortalité infantile en pays de colonisation}

\section{Christiane Robitaille}

Volume 16, numéro 2, printemps 2004

Deuil, blessure vive

URI : https://id.erudit.org/iderudit/1074109ar

DOI : https://doi.org/10.7202/1074109ar

Aller au sommaire du numéro

Éditeur(s)

Université du Québec à Montréal

ISSN

1180-3479 (imprimé)

1916-0976 (numérique)

Découvrir la revue

Citer cet article

Robitaille, C. (2004). La mortalité infantile en pays de colonisation. Frontières, 16(2), 9-16. https://doi.org/10.7202/1074109ar

\section{Résumé de l'article}

En recueillant les témoignages des membres de sa famille, l'auteure a été en mesure de documenter le vécu d'une famille de l'Abitibi affligée par le décès de plusieurs nouveau-nés et jeunes enfants. Elle interroge ces données pour mieux comprendre le travail de deuil. d'utilisation que vous pouvez consulter en ligne.

https://apropos.erudit.org/fr/usagers/politique-dutilisation/ 


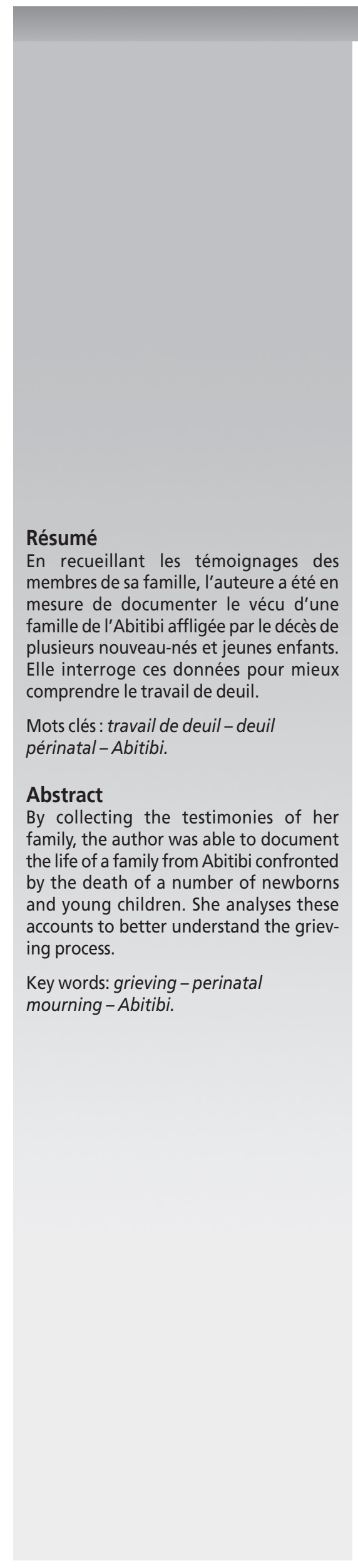

\section{La mortalité infantile en pays de colonisation}

Christiane Robitaille,
cinéaste.

Dans ma famille, les références à la mort et surtout à la mortalité infantile ont fortement imprégné les récits que mes parents ainsi que mes frères et sœurs aînés me faisaient de la vie en terre de colonisation. Mon père, dans sa jeunesse, avait souffert de peur panique à la vue d'un cadavre. Ma mère, aujourd'hui décédée, a mis au monde dix-sept enfants, dont six sont morts peu après la naissance ou en bas âge.

J'ai effectué une recherche auprès des membres de ma famille au sujet des pratiques et des discours ayant entouré la mort de ces enfants. J'ai interrogé mes aînés afin d'obtenir les récits de ces événements. À mesure que mon enquête avançait, je me suis sentie fortement interpellée par ces récits. Comme le souligne Magali Uhl (2002, p. 46), lorsqu'il est question de la mort, la relation de recherche est « une relation de sujet à sujet et non plus de sujet à objet ». Il s'agirait, selon elle, de rapports de «réciprocité dialectique ». J'ai effectivement été confrontée à mes propres deuils, en particulier à celui de mon enfant, mort de façon soudaine à l'âge de quatre mois alors qu'il était chez sa gardienne. J'ai pris conscience que l'objet de ma recherche avait un lien avec mon histoire personnelle : la perte de cet enfant m'a semblé pendant longtemps relever du deuil impossible, alors que ma mère avait su traverser tous ses deuils, même si certains avaient été particulièrement douloureux.

Ma recherche s'est donc transformée et les questions qui se sont imposées à moi furent celles-ci : comment, après toutes ces pertes, ma mère avait-elle pu continuer de mettre au monde des enfants, dans le contexte de la maternité obligatoire, dans des conditions de vie très rudes, loin des ressources médicales et des soins nécessaires? Comment avait-elle, quoique sa santé physique en fût altérée, traversé ces épreuves en conservant sa santé mentale? En un mot, comment avait-elle fait son travail de deuil ? Comment mes frères et sœurs, qui étaient des enfants d'âges différents au moment de ces morts d'enfants, avaient-ils « métabolisé ${ }^{~}$ » ces deuils ? Comment s'inscrivaient les enfants morts dans la constellation familiale?

En plus des récits que j'ai pu recueillir auprès de mes aînés, je me suis remis en mémoire certaines conversations avec ma mère. J'y ai retrouvé les récits qu'elle me faisait. Au fil de ma recherche, ma mère a repris vie, si je puis dire. En effet, il m'a semblé qu'à travers lectures et entrevues, réflexions et rêves personnels, s'est engagée une sorte de conversation imaginaire avec elle, conversation à travers laquelle s'est effectué pour moi un réel travail de deuil, deuil de ma mère et deuil de mon enfant. 


\section{LES RAVAGES DE LA MORTALITÉ INFANTILE DANS UNE FAMILLE DE L'ABITIBI, DE 1926 À 1947}

Je suis la quinzième d'une famille de dix-sept enfants, six morts en bas âge, onze vivants. Je suis née en région de colonisation et j'y ai exercé la profession d'infirmière pendant plusieurs années. J'ai donc été amenée à prodiguer des soins aux personnes en phase terminale, à me questionner sur l'accompagnement des mourants et sur les questions de deuil en général. De plus, comme je l'ai déjà mentionné, j'ai perdu moi-même un bébé de façon tragique à l'âge de 25 ans.

Mes interlocuteurs ont été quatre de mes aînés qui ont en ce moment entre 75 et 60 ans : mon frère Pierre a 75 ans; mes sœurs sont Michelle, 72 ans, Thérèse, 70 ans et Beth qui a 61 ans. J'indiquerai plus loin leur âge au moment du décès de chacun des bébés. Ma mère aurait aujourd'hui 98 ans; mon père en aurait 102.

J'ai d'abord fait une première démarche par téléphone pour sonder le terrain. Étant donné qu'il s'agissait de deuils vécus dans l'enfance, je craignais de réveiller des émotions perturbatrices et de déclencher des refus. La réaction de mon frère et de mes sœurs ayant été tout à fait positive, je me suis donc rendue à leur domicile. Je leur avais fait connaître à l'avance, à leur demande, mes trois principales questions tout en leur demandant de ne pas se préparer, car je désirais des récits. J'ai tenté une approche dite semi-directive à partir d'entretiens focalisés (Blanchet, 1985, p. 51) en recherchant des récits et en explorant avec mes interlocuteurs l'ensemble des thèmes, en demandant des précisions ou des détails, en impliquant mes propres souvenirs, en reformulant et en explorant le contexte avec eux. Toutefois, mon frère aîné m'a demandé de lui fournir à l'avance toutes les questions, car il voulait "alimenter sa mémoire». Cette entrevue a donc été faite de manière un peu plus directive. J'ai enregistré les entrevues sur cassettes et j'en ai tiré des données par la suite.

Le climat de ces entrevues était, bien sûr, chargé d'émotions, car nous n'avions que rarement parlé de ces événements sauf parfois en présence de notre mère. De plus, la mort de mon fils ayant été peu abordée avec mes frères et sœurs, je craignais un certain malaise. J'ai donc soulevé moimême cette question au début des entrevues en soulignant le fait que j'avais mis des années à faire ce deuil. Maintenant que je pouvais en parler, je désirais en savoir plus sur les morts d'enfants dans notre famille.

J'ai fait un résumé des entrevues tout en essayant de garder le ton et le vocabulaire utilisés par mes interlocuteurs. J'ai retenu les données concernant les circonstances des agonies et des morts, tout en étant attentive aux données concernant le climat familial par la suite, ceci pour alimenter mon questionnement sur le travail de deuil.

Voici des extraits de ce que m'ont raconté ma mère, mon frère Pierre et mes sœurs Michelle et Thérèse au sujet de la mort de mes cinq frères, Irénée, Arthur, Ange-Albert, Gérard et Yvan, et de ma sœur Andréanne.

Irénée était le premier enfant de mes parents. Ma mère avait alors 22 ans. Il est né le 4 septembre 1926 et il est mort le 14 septembre.

Il y a quelques années, ma mère, qui avait alors 73 ans, m'avait raconté ce qui s'était passé en ces termes: "Lors de l'accouchement, j'ai crié pendant trois jours. Ton oncle Gérard m'entendait de l'étable! L'enfant ne passait pas, il était trop gros. J'ai eu une déchirure complète. Il pesait neuf livres... ils ont mis les forceps... il est mort 10 jours après sa naissance. »

Je suis la personne qui possède le plus d'informations sur ce décès parce que j'ai questionné ma mère. De l'impact de ce décès, ma mère m'a dit peu de chose. Sauf que sa manière de le raconter, l'émotion contenue dans son récit m'ont fait ressentir que pour elle, la naissance et la mort de cet enfant ont été comme une seule et même effroyable douleur. Une photo existait, que je n'ai pu retrouver, qui montrait l'enfant sur les planches dans sa robe de baptême. Cette photo était rangée parmi les photos des vivants, dans les affaires personnelles de ma mère, et je me souviens l'avoir questionnée à ce sujet alors que j'avais sept ou huit ans. Elle me répondait succinctement: "C'est le petit Irénée, mort petit bébé. »

Ce premier deuil a sûrement été une terrible épreuve et un choc. Ma mère a accouché d'un deuxième enfant le 12 novembre 1927, mon frère Pierre, qui est toujours vivant.

Arthur est né le 28 décembre 1928 et il est décédé le 14 janvier 1929. Ma mère avait alors 24 ans. Mon frère Pierre pense qu'il avait la jaunisse. Je n'ai pu recueillir aucun récit à son sujet. Personne ne sait.

Ange-Albert est né le 5 juin 1933. Il est décédé en mars 1934. Ma mère avait 30 ans et elle était enceinte. La famille comptait alors trois enfants.

Mon frère Pierre, qui avait sept ans à l'époque, se souvient que toute la famille souffrait alors de la grippe : "L'enfant était malade, nous avions tous la grippe. Maman avait écrit au docteur et avait remis la lettre au postillon qui allait chercher la poste aux deux jours, à cheval, au village voisin, à neuf milles. Il devait ramener des médicaments, mais l'enfant est mort avant. »

Mon père n'était pas à la maison lors du décès d'Ange-Albert. Pierre se souvient de plusieurs femmes qui ont aidé ma mère :
Maman nous a réveillés dans la nuit pour nous dire que le bébé est décédé. La voisine d'en face était là, madame Ovide, elle avait veillé avec maman toute la nuit. Maman était enceinte d'Agnès. Père était dans le bois, sur le lac Abitibi. Quelqu'un est allé le chercher, il est arrivé le soir du même jour ou le lendemain du décès. Maman pleurait de manière silencieuse. Une autre voisine, madame Berthiaume, a fait la toilette mortuaire. L'enfant a été exposé quelques jours sur le moulin à coudre, devant la fenêtre du camp. On avait déposé un drap blanc sur le moulin. Oncle J. a construit un petit cercueil et tante L. a mis du brocart blanc à l'intérieur. Les voisins sont venus rendre visite, oncles et tantes aussi. Les deux voisines, madame Cyril et madame Rose B., se sont occupées de nous, elles nous ont fait manger.

Pierre m'a parlé de sa perception de la mort en disant:

Je savais ce que c'était la mort parce que je prenais des lièvres au collet et nous les mangions, cela faisait partie de la subsistance quotidienne. À ce moment-là, tu sais que la mort, c'est la fin de la vie. Pour moi, c'était mon frère et il était décédé de maladie. Un événement fatal, on ne peut rien faire, on ne peut contrer. Cela se vivait avec un certain fatalisme, en ce sens que c'était accepté, c'étaient des choses pour lesquelles on ne pouvait rien. C'était la foi du charbonnier, décision de la Providence.

Mon frère Pierre se souvient aussi des réactions de ses sœurs plus jeunes :

Michelle a été la plus affectée car elle le berçait! Maman avait monté la couchette dans l'entretoit, là où elle rangeait la nourriture. Thérèse montait derrière elle et allait taper sur le matelas en disant: " n'as pu timomon!» La vie a continué. Naturellement, perdre un enfant, pour une mère, c'est terrible, mais il fallait manger, aller tirer la vache, rentrer l'eau, autrement dit, il fallait se débrouiller avec sa peine. On sentait, plus qu'on voyait la peine.

Ma sœur Michelle, qui avait alors quatre ans, dit avoir tout oublié. Ma sœur Thérèse avait deux ans. Lorsque je lui ai parlé de la mort d'Ange-Albert, elle m'a dit :

Je n'avais pas de souvenir d'AngeAlbert, mais quand Pierre a raconté à mon anniversaire qu'à deux ans j'allais taper sur le matelas, je me suis sentie mal... et par la suite toute la semaine. 
Cela a réveillé des choses enfouies, des choses indéfinissables... On ne tient pas compte de la douleur d'un enfant. J'ai l'impression d'avoir été très marquée... Il s'est produit quelque chose entre moi et maman... Maman disait souvent: "Il n'en serait pas mort, cet enfant-là, si j'avais pu le faire soigner!» Ce n'était pas drôle d'entendre ça! Elle n'acceptait pas la pauvreté et le dénuement qui lui avaient enlevé cet enfant-là. Maman a porté cette peine toute sa vie. Maman m'a raconté que tante Marie (la sœur de la mère de ma mère, qui avait semble-t-il un retard mental, et qui avait remplacé la mère de maman après sa mort) avait dit: "Il est trop beau, cet enfant-là, il ne vivra pas!» Vois-tu, c'est toutes des choses... la tante Marie avait dit ça!

Andréanne, née le 8 juin 1937, est décédée à la suite d'une maladie en août 1937. Ma mère avait alors 32 ans.

Selon mon frère Pierre, Andréanne aurait souffert d'une longue maladie: "J'avais 10 ans. Je me souviens parce que maman avait téléphoné au docteur, une ligne téléphonique venait d'être construite, et il avait envoyé des médicaments par le facteur. Je guettais le facteur. Elle a été longtemps malade, elle est morte comme un petit poulet. »

Ma sœur Michelle se souvient des derniers moments d'Andréanne :

J'avais huit ans. Le bébé avait une diarrhée constante. Le jour de son agonie, maman l'avait couchée sur un oreiller sur la table de cuisine. Papa était absent. Maman et madame Boutin, la voisine, se relayaient pour la veiller. Maman nous a dit: "Venez voir votre petite sœur, le Bon Dieu va venir la chercher. » Nous étions autour de la table, avec les plus petits dans les bras. Il y avait une atmosphère de recueillement. Maman a pris de l'eau bénite et elle l'a signée en faisant un signe de croix. Maman avait bien de la peine, cela faisait mal au cœur. Maman disait que c'était devenu un ange, un petit ange au ciel. Elle disait ça de tous ses enfants morts.

Gérard, né le 24 février 1940. Mort-né. Ma mère avait 36 ans. Le plus âgé des enfants avait alors 13 ans.

Selon mon frère Pierre, Gérard est décédé alors que notre famille, qui habitait précédemment dans un rang, venait d'emménager au village. Il semble qu'il ait alors été plus facile d'avoir accès à un médecin :

J'avais 13 ans. On venait de déménager à l'été au village. Le docteur Balthazar était venu pour l'accouchement. Madame Doyon, la nouvelle voisine, avait assisté à l'accouchement et avait ondoyé l'enfant. Madame Béland, l'autre voisine, avait pris soin des enfants. Le médecin avait couché à la maison, car c'était une question de vie ou de mort pour notre mère.

Ma sœur Michelle, l'aînée des filles, a été grandement effrayée de constater que cette naissance mettait ma mère en danger :

J'avais 10 ans. Maman m'a réveillée et m'a dit d'essuyer le sang par terre. J'ai eu très peur qu'elle meure. Elle faisait une hémorragie. L'enfant n'était pas à terme. Madame Doyon avait mis l'enfant sur le bureau, enveloppé dans des draps blancs. Papa était absent. Il est revenu du bois. Je le vois encore arriver, avec des glaçons dans sa barbe. Je crois que maman avait trop travaillé, avec le déménagement et la besogne... Maman avait, par la suite, peur de perdre ses autres enfants. Elle avait toujours une peine immense. Mais j'ai l'impression que ces enfants demeuraient proches d'elle. Elle gardait un contact spirituel, elle leur demandait de l'aide.

Ma sœur Thérèse n'a gardé que de vagues souvenirs de la mort de Gérard et elle considère qu'on ne lui a pas vraiment parlé de ce qui se passait alors : "J'avais huit ans. Sur le chiffonnier, on voyait une affaire... une serviette par-dessus, comme une petite tête... maman n'a pas parlé, n'a pas dit que c'était un petit frère qui était mort. »

Yvan est né le 26 juillet 1943 et il est mort le lendemain ou le surlendemain, pendant le trajet de retour de son baptême. Ma mère avait alors 39 ans.

L'accouchement d'Yvan aurait été provoqué par un accident, selon le récit de mon frère Pierre:

J'avais 16 ans. Je faisais le train avec maman. Une vache l'avait ruée.

Maman était tombée dans la merde de vache. Elle m'avait demandé de l'aider à se relever. Le lendemain matin, l'enfant était né. Papa était absent. Le docteur est venu. À la demande de papa, je suis allé creuser la fosse au cimetière. Des voisines étaient là. Je me souviens moins bien, j'étais souvent à l'extérieur de la maison. Papa camouflait ses émotions, surtout devant nous autres, se montrait stoïque. Maman, je ne dirais pas qu'elle cachait sa peine, elle la vivait, mais c'était une peine intérieure.

À cette époque, tout le monde jugeait nécessaire que le baptême d'un nouveauné ait lieu le plus rapidement possible après la naissance. Ma sœur Michelle se souvient du jour du baptême d'Yvan, qui est devenu un jour de deuil :

J'avais 13 ans. Maman avait dit qu'il lui semblait malade. Le curé était absent. La voisine l'a habillé de sa robe de baptême et ils sont partis en camion pour la ville voisine avec papa. Il est décédé dans le camion au retour. C'était le lendemain ou le surlendemain de sa naissance. Maman pleurait avant le départ, comme si elle avait eu un pressentiment... elle se reprochait ensuite de ne pas l'avoir ondoyé. L'avait-on nourri avant son départ? Je me demande... on devait l'avoir fait...

Le récit de ma sœur Thérèse, qui avait alors 11 ans, parle aussi de cette fête qu'aurait dû être le baptême d'Yvan : «Il aurait fallu qu'il reste à la maison. Monsieur Béland avait acheté un sac de bonbons pour fêter le baptême. Il savait plus quoi faire avec ses bonbons, c'était censé être pour une fête. Mais l'enfant était mort! »

Confrontée à tant de naissances si souvent suivies d'un décès, Thérèse se souvient qu'elle avait élaboré une sorte de théorie lui permettant de prévoir si la mort allait ou non frapper :

Maman était toujours triste, avec une grosse besogne sur le dos. Nous autres, on était bien impressionnés par ça. Je faisais des pronostics : un, c'est le tour à vivre; un, c'est le tour à mourir. Ça ne marchait pas toujours: Mario, j'ai été bien étonné qu'il vive... Après Yvan, c'était toi ; c'était ton tour à vivre... Tu sais ça se passait de même dans les familles... donc la peine était moins grande... mais j'étais impressionnable... cela faisait un millefeuilles d'émotions, je dirais un mille-pattes dans la poitrine...

Ma sœur Thérèse se souvient aussi de la peine de papa lors du décès d'Yvan : «Papa était écrasé, dépassé, il se sentait impuissant. Il était à terre... il trouvait son réconfort dans la prière... il était soucieux avant d'aller prier et à la fin de la prière il devenait beau...»

Pour compléter ces récits au sujet du décès de mes cinq frères et de ma sœur, j'ajoute ici la description d'un autre événement qui m'a été raconté lors des entrevues. Cela s'est passé en février 1947, lorsque ma sœur Éliane, née le 22 février, a dû recevoir l'extrême-onction à la maison deux jours après sa naissance parce qu'elle était très malade. Éliane a survécu. Ma mère avait alors 43 ans et j'avais deux ans et demi.

Ma sœur Thérèse raconte ce qui s'est passé : 
J'avais 15 ans. Je relevais maman.

Éliane n'avait que deux jours. Elle est devenue bleue, bleue... j'ai attelé le chien et je suis allée voir le curé... Il disait sa messe... Je lui ai dit: «Vite, vite, le petit bébé va mourir!» Il a répondu: "Je finis ma messe et je viens. » Je le vois encore venir, avec sa grande mante noire qui se soulevait. Il s'en venait comme un oiseau... Il a dit: "Mettez-la sur un oreiller, la tête en bas. Il lui a donné l'extrême-onction et il est parti à la ville, chez le docteur R. Il est revenu le soir même avec les médicaments... il avait des connaissances médicales... Le curé venait comme un sauveur...

$\mathrm{Tu}$ vois le climat dans lequel...

tu vois ta mère devant ça... ah, misère... elle aurait pu mourir dans la journée... l'impuissance!

Cette intervention du curé est aussi restée gravée dans la mémoire de ma sœur Beth, qui en a fait spontanément le dessin à la suite de notre entretien. Elle avait gardé une image très nette de ce moment, même si elle n'avait que cinq ans lorsqu'elle a vécu cet événement. Le tableau représente le moment où la dernière-née, Éliane, étant sur le point de mourir, reçoit l'extrêmeonction. J'y suis représentée à genoux, âgée de deux ans et demi, ainsi que les autres membres de la famille qui étaient présents. Ma mère est assise sur le lit, dans la chambre, tenant l'enfant dans ses bras. L'officiant est bien identifiable, car il a sur la tête une barrette et il tient une croix à la main.

Beth décrit la scène:

J'avais 5 ans. Je vois une image. Je vois le curé dans l'embrasure de la porte... Maman avait le petit bébé, Éliane, petite, petite, maigre et bleue... On était à genoux... Le curé avait son espèce de coffre avec de l'huile... Maman, énervée, avait dit: «Va chercher le curé. » Maman était assise sur le bord du lit et quelqu'un, peutêtre toi, répétait comme un enfant inquiet: "Qu'est-ce qu'il fait, qu'est-ce qu'il fait? » Je ne savais pas vraiment ce qui se passait, on ne m'avait pas expliqué... Le soir, on avait mis la couchette derrière le poêle à bois... Le curé était revenu le soir avec les médicaments.

Ces événements sont survenus après la Première Guerre mondiale, en temps de crise et pendant la Seconde Guerre mondiale. Les conditions de vie en région de colonisation étaient alors très dures; pas d'électricité, pas d'eau courante, dans une région de grands froids. Il n'y avait pas d'hôpitaux dans la région; les médecins en place devaient parcourir de grands territoires; les vaccins contre les maladies contagieuses n'avaient pas encore été mis au point. Les naissances avaient lieu à la maison, parfois sans assistance médicale, mais avec l'aide d'une sage-femme ou d'une voisine. Les mortalités avaient aussi lieu à la maison, entourées des proches et souvent avec l'assistance et le support de la même personne qui avait aidé à l'accouchement. Dans les récits que j'ai recueillis, la présence des voisines est toujours signalée. Malheureusement, mon père est absent lors des décès. $\mathrm{Ce}$ sont les voisines qui supportaient la femme en deuil, qui lui apportaient aide et réconfort. Cette solidarité féminine est un élément important à considérer dans la résolution des deuils en milieu rural, car la mort de tous ces enfants vécue dans la solitude aurait sans doute ajouté à la détresse des femmes qui perdaient leurs enfants.

\section{LES DEUILS DE MA MÈRE}

Dans le Québec rural des années 1920 à 1950, les pratiques et discours étaient fortement tributaires de la vision véhiculée par la religion catholique concernant la vie et la mort. Le clergé catholique insistait fortement sur l'obligation d'accepter tous les enfants que Dieu vous donne. En effet, à l'époque où ma mère a donné naissance à ses enfants, les femmes des milieux ruraux, (probablement plus que celles des milieux urbains où la religion avait moins d'emprise) vivaient l'obligation de la maternité. Selon les préceptes de l'Église catholique, ces femmes n'avaient pas le droit de « refuser la famille », c'est-à-dire qu'elles n'avaient pas le droit de refuser une relation sexuelle avec leur conjoint, sous peine de péché mortel. Les naissances n'étaient donc pas toutes désirées, et de plus, l'état de santé de ces femmes se ressentait de ce «travail à la chaîne ».

En examinant les données que j'avais recueillies lors des entrevues avec mon frère et mes sœurs, je me suis demandé si ma mère avait été en mesure de faire un travail de deuil. Michel Hanus (2000, p. 28) utilise l'expression travail de deuil pour désigner «le travail psychologique intérieur nécessité par toute perte de quelque importance, travail nécessaire pour intégrer cette perte». Il distingue trois phases dans l'état deuil : le début, qui constitue un état de choc, une période centrale et une terminaison. 1) L'état de choc intéresse l'ensemble de la personne et s'exprime sur tous les plans: santé physique, vie affective, relations sociales. Les émotions ressenties sont un mélange d'abattement et de manifestations émotionnelles aiguës dont la révolte et la colère. 2) La période centrale se caractérise par un état dépressif réactionnel où la souffrance intérieure s'accompagne d'une extrême fatigue et de grandes difficultés de fonctionner normalement. Hanus (2000, p. 30) écrit à ce sujet: "Lorsque nous sommes en deuil, il ne nous paraît pas possible de nous accorder des satisfactions.» 3) Le deuil évolue par la suite vers une terminaison, mais il ne se finit jamais complètement. "C'est d'abord dans les rêves que l'endeuillé constate qu'il s'intéresse à de nouveaux projets, de nouvelles personnes» (Hanus, 2000, p. 30).

À l'intérieur de ces phases du deuil, trois processus agissent en nous inconsciemment; selon Michel Hanus, ces processus constituent l'essence même du «travail de deuil ». 1) Il s'agit surtout de la reconnaissance de la réalité psychique entraînée par la perte ; il se fait un travail d'acceptation à travers un état de déséquilibre provisoire et à travers l'émergence des sentiments violents et contradictoires qui nous amènent à accepter l'impuissance. 2) Il se produit ensuite un renforcement de l'intériorisation de la relation avec l'être que nous aimons et que nous venons de perdre. Ne pouvant rejoindre cette personne aimée dans la réalité extérieure, nous pouvons retrouver en nous sa présence intérieure. L'essentiel du travail de deuil se trouve dans cette remémoration qui permet de donner une autre dimension à notre relation d'amour. 3) Le troisième processus est un travail sur les sentiments inconscients de culpabilité. Cette culpabilité est sans relation avec la réalité objective; elle est en relation avec notre ambivalence intérieure qui existe dans toutes nos relations les plus chères. Ces sentiments inconscients s'épuisent progressivement par la souffrance que nous vivons et par les inhibitions que nous nous imposons en temps de deuil.

Le premier enfant de ma mère, Irénée, est décédé à la naissance. Ma mère avait alors 22 ans. Aurait-elle fait une dépression post-partum ? À l'époque, la dépression post-partum était rarement diagnostiquée, à moins qu'il ne s'agisse d'une psychose post-partum. Mes expériences d'infirmière auprès des femmes nouvellement accouchées, ainsi que la façon dont ma mère me racontait ces événements, me portent à le croire. Cela n'a jamais été nommé ni raconté dans ma famille, même pas par ma mère. Sauf que Thérèse m'a dit que ma mère semblait dépressive après ses accouchements. Je ne crois pas que le deuil d'Irénée soit devenu ce que Michel Hanus appelle un "deuil compliqué», car le fait qu'elle accouche d'un autre enfant le 12 novembre 1927 indique qu'elle aurait été enceinte en février 1927. Or ma mère me disait que, quand elle était enceinte, elle se refaisait une santé. 
Les « deuils compliqués » se manifestent, selon Hanus, dans trois directions: dans une dépression chronique, dans la prise inconsidérée de risques et dans la dégradation de la santé. Il explique ces complications par l'inflation de sentiments inconscients de culpabilité, en relation avec une ambivalence excessive :

Sur le plan psychopathologique, ces formes de complications relèvent tout à la fois du besoin de s'identifier à celui qui n'est plus là avec le sentiment inconscient de le conserver par nos souffrances, de la difficulté à accepter qu'il soit vraiment mort, et d'une contrainte à se faire souffrir en punition de notre ambivalence. (Hanus, 2000, p. 35)

Je crois cependant que la perte de l'enfant Ange-Albert, survenue sept ans plus tard, a laissé ma mère dans un état de grande fragilité psychologique. Encore à l'âge de 60 ans, l'évocation de cet enfant faisait jaillir ses larmes... Je l'ai constaté moi-même à plusieurs reprises. Sa santé s'est-elle dégradée à partir de ce momentlà, ce qui serait un signe, selon Hanus, d'un deuil compliqué ? Il se peut qu'elle ait vécu un moment de franche dépression, ce qui est plausible et compatible avec le fait que ma sœur Thérèse, alors âgée de deux ans, ait senti remonter en elle "des émotions indéfinissables » lorsque mon frère Pierre lui a raconté qu'elle «allait taper sur le matelas » du bébé décédé. Un enfant de deux ans ne peut nommer son malaise, mais il peut éprouver un sentiment d'abandon si la mère devient absente à ses autres enfants à cause d'un état dépressif. Il peut aussi, en raison de son lien avec la mère, ressentir les émotions qui la traversent. Des études concernant le lien télépathique entre mère et enfant dans la petite enfance semblent démontrer que ce lien existe (Ehrenwald, 1981).

Si la santé de ma mère s'est dégradée, cela peut fort bien avoir été les conséquences de toutes ses grossesses répétées, lesquelles s'ajoutaient aux deuils d'enfants. Mon frère Pierre m'a dit qu'il a toujours vu ma mère enceinte. Il est certain qu'après toutes ses épreuves, la santé de ma mère s'est altérée. En témoignent certaines photos, alors qu'elle avait 47 ans, qui la montrent d'une maigreur extrême. Dans ma famille, on emploie l'expression : « Elle avait l'air d'une déterrée ! » Cette expression est en résonance directe avec l'idée de la mort ! L'obligation de devoir faire ce travail de fabrication d'enfants à la chaîne avait certainement épuisé ses réserves vitales.

\section{LES RESSOURCES DE MA MÈRE}

Toutefois, même si sa fragilité m'apparaît évidente à certains moments de sa vie, ma mère a été une personne aimant la vie et aimant ses enfants. Elle s'est même remariée à l'âge de 72 ans, étant tombée en amour comme une jeune fille. Je crois qu'elle a puisé une grande force dans les croyances de sa culture chrétienne, croyances dont elles nous faisaient part, en particulier celles-ci : l'idée d'un Dieu bon qui se manifeste par la Providence ; la croyance que ses enfants morts, devenus des anges au ciel, ainsi que sa mère morte la protégeaient; un recours à sainte Anne, médiatrice des femmes mères.

La religion catholique ne présentait pas beaucoup de modèles de femmes mères, sauf celui de la Vierge ayant conçu avec l'opération du Saint-Esprit, mais sainte Anne avait enfanté, de plus, d'une fille. Pour ma mère, c'était un modèle important, d'autant plus que huit filles ont survécu parmi toutes ses grossesses à risques.

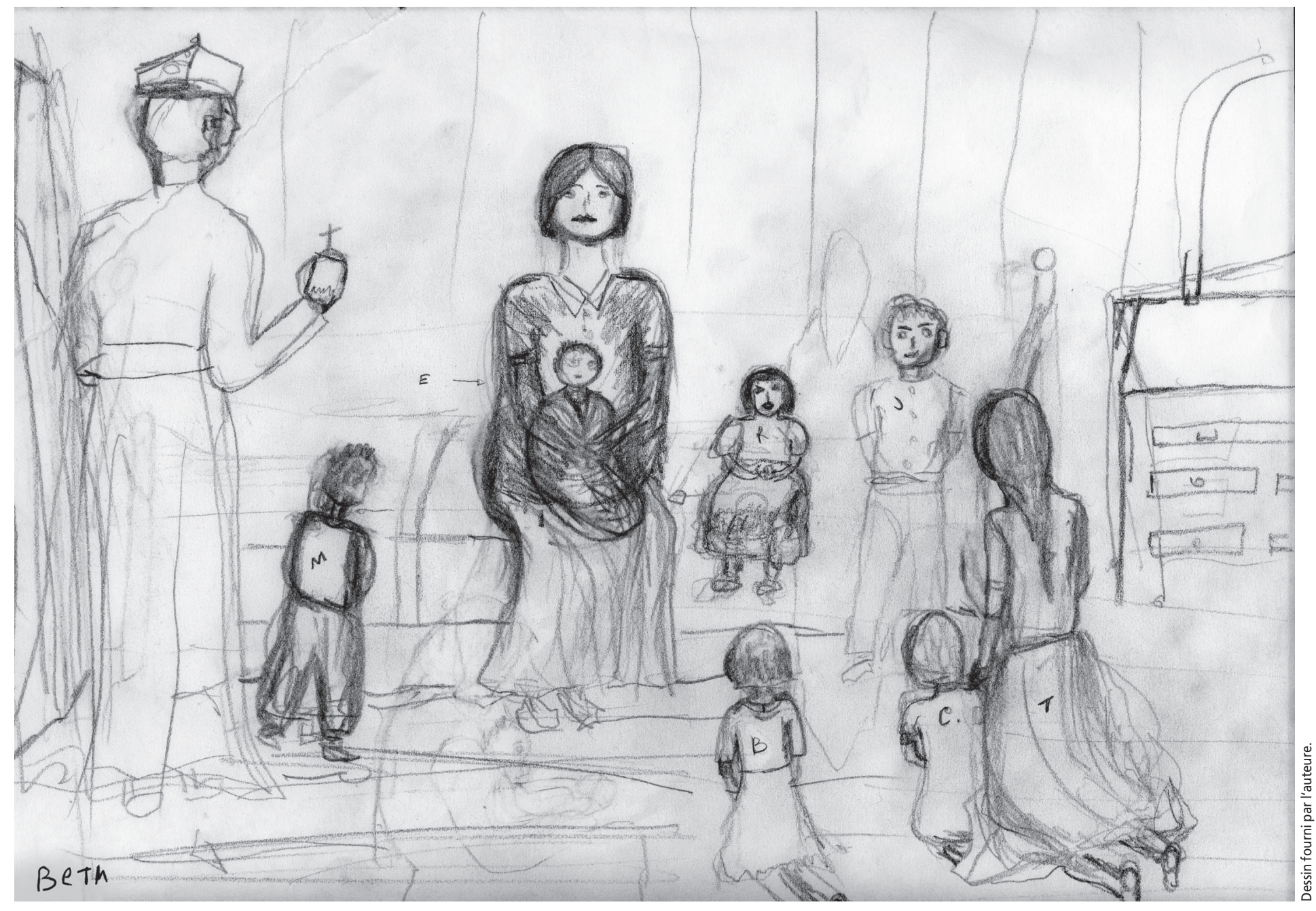


De plus, contrairement à mon père qui avait fait des études pour la prêtrise et dont la foi était celle d'un initié, ma mère avait une attitude envers la religion qui relevait plutôt d'une croyance au merveilleux : par exemple, elle me racontait que des lutins avaient tressé la crinière des chevaux durant la nuit, et cette croyance était, pour elle, mise sur le même pied que la croyance aux anges du ciel. Elle croyait aux puissances de l'invisible. Cet invisible était parfois malicieux, comme cette histoire des lutins, mais il était le plus souvent dispensateur de bienfaits. Je crois que cette attitude lui venait de ses origines beauceronnes, région du Québec riche en contes et légendes. Elle avait certainement entendu raconter des récits de ce genre.

Un autre aspect de sa personnalité lui a permis, je crois, de traverser toutes ces difficultés : elle était une personne créative. En effet, réussir à sauver des enfants, leur donner des soins, entretenir avec eux une relation qui leur permette de grandir, tenir maison, faire en sorte que la chaleur et le bien-être soient présents, toutes ces attitudes qui entretiennent la vie prenaient leur source dans sa créativité. Elle possédait aussi une grande habileté manuelle, et aucun des métiers artisanaux n'avait de secret pour elle : couture, broderie, tricot, dentelle. Je me souviens avoir porté un chandail pour lequel j'avais assisté à toutes les étapes de production: de la laine tondue sur le dos du mouton, à l'écheveau teint dans une grande bassine sur le poêle, à la belle couleur rouge qui est apparue à travers les fumées et l'odeur d'ammoniaque, pour finir sur des broches à tricoter avec lesquelles j'ai modestement fait quelques mailles à l'endroit. On pourrait croire que ces activités étaient motivées par la seule nécessité, mais à voir le visage de ma mère s'éclairer quand elle manipulait des tissus en disant : "C'est du beau, c'est du beau! », il était évident que la joie et le plaisir de créer animaient ses traits.

Faisant référence à la «fonction de réparation de l'objet et du sujet » que développe Mélanie Klein, je crois que c'est grâce à sa grande créativité que ma mère a réussi à «réparer ses objets d'amour» et à "se réparer elle-même en tant que sujet ». Les notions d'objet et de sujet font référence ici à la théorie psychanalytique développée par Mélanie Klein selon laquelle la vie fantasmatique est présente chez l'enfant dès sa naissance. Elle utilise la notion « d'objet intérieur » créé par les fantasmes. "L'objet interne » est d'abord un fantasme projeté après coup sur l'objet réel. La notion de «sujet» inclut la notion de subjectivité, "l'être capable de pensée, considéré comme le siège de la connaissance » (Niobey, 1986, p. 681).
Pour Mélanie Klein, les actes créateurs ont une fonction réparatrice. Selon Chasseguet-Smirgel, Mélanie Klein distingue deux catégories d'actes créateurs: ceux qui concernent la réparation de l'objet d'amour et ceux qui concernent la réparation du sujet lui-même. Chasseguet-Smirgel (1971, p. 103) explique ce qu'est la création : «C'est une impulsion profonde du désir de pallier, par ses propres moyens, les manques laissés ou provoqués par autrui. L'acte créateur constitue l'une des modalités privilégiées d'accomplissement réparateur. »

Mélanie Klein aurait, au début, situé les racines de la sublimation et de la créativité dans le sentiment de culpabilité lié à la position dépressive et au désir corollaire de réparer l'objet d'amour censé avoir été abîmé par le sujet. Cependant, par la suite, elle émet l'opinion que c'est la relation au sein maternel qui est source de créativité :

Il s'agit en effet, de la relation au sein maternel conçu comme source de vie et manifestation de la créativité. Le désir d'intérioriser la source de vie et de s'identifier à elle, en possédant ainsi à jamais de quoi étancher sa soif et celle des autres, en s'affranchissant par là même de l'objet, assurer sa propre complétude, fonder sa toute-puissance, deviennent alors la base à mon sens, de toute créativité, pour peu que l'on poursuive, jusqu'à ses développements ultimes, la pensée de Mélanie Klein. (Chasseguet-Smirgel 1971, p. 105)

Est-ce l'identification à sa mère, morte alors qu'elle avait quatre ans, qui lui a permis, pour reprendre les propos de Janine Schasseguet-Smirgel « d'intérioriser la source de vie et de s'identifier à elle dans une relation au sein maternel conçu comme source de vie et manifestation de la créativité»? J'en ai l'impression. Ma mère a certainement été victime de la misogynie catholique à travers une sexualité obligée. Toutefois, je crois qu'elle a quand même, en partie, détourné à son profit ce grand courant d'énergie venu d'une créativité féminine et qu'elle a, comme bien des femmes de son époque, traversé le siècle grâce à ce courant souterrain.

Le travail de remémoration qui se produit vers la fin du travail de deuil me semble aussi être un travail de réparation de l'objet d'amour perdu. Selon Hanus (2000, p. 30) : «Il se produit un renforcement de l'intériorisation de la relation avec l'être que nous aimons et que nous venons de perdre. L'essentiel du travail de deuil se trouve dans cette remémoration qui permet de donner une autre dimension à notre relation d'amour. »

Dans l'élaboration d'une relation intérieure avec ses défunts et par la mise en œuvre de toutes ses ressources créatrices, ma mère semble donc avoir fait un travail de deuil.

\section{LES EXPÉRIENCES DE DEUIL VÉCUES PAR MON FRÈRE ET MES SCEURS}

J'ai peu de données sur l'état psychologique dans lequel se trouvaient mes frères et sœurs lorsque se sont produits les décès relatés ici. Je ne peux qu'émettre quelques hypothèses à la lumière des propos de Michel Hanus.

Selon Hanus, le deuil est plus difficile chez l'enfant : "du fait que ses énergies sont consacrées à son développement, une partie du travail de deuil sera toujours remise à plus tard, au sortir de l'adolescence ». C'est vers quatre ans qu'en général les enfants commencent à comprendre que la mort est irréversible, mais il leur faudra du temps avant de pouvoir l'accepter. Avant l'âge de quatre ans, la mort n'est «ni naturelle (on ne meurt pas, on est tué) ni irréversible. Elle est contagieuse ».

Le deuil des enfants comporterait les mêmes phases que celui des adultes sauf que l'état dépressif de l'enfant se manifestera surtout dans le comportement et pourra affecter sa santé. Un deuil dans l'enfance est toujours un traumatisme, mais ses conséquences ultérieures sont variables, car tout dépend de la relation qui existait auparavant avec le décédé. Michel Hanus insiste sur le fait que l'accompagnement des enfants est nécessaire surtout si la perte se situe au niveau affectif et influe sur son mode de vie (par exemple lorsque la mort de la mère entraîne le placement en foyer nourricier).

Les données recueillies auprès de mon frère et de mes trois sœurs me portent à croire que leur travail de deuil a été difficile. Il n'y avait pas de déni, mais on parlait peu de la mort aux enfants. Mon frère Pierre le signale ainsi lors du récit de la mort d'Ange-Albert : «Il fallait se débrouiller avec sa peine. » Il ne semble pas qu'il ait trouvé d'écho à ses questionnements ni que l'on ait encouragé l'expression de ses émotions. «Les enfants modèlent leur comportement sur celui des adultes, et ne pourront manifester leurs sentiments et leurs émotions que si on le leur permet» (Hanus, 2000, p. X). Mon père se montrait stoïque et camouflait ses émotions, selon mon frère. Alors son fils se devait probablement de l'imiter. De là à développer l'idée d'un certain fatalisme... mais les enfants se sentent toujours coupables de la mort d'un de leurs proches, selon Hanus. Il a dû être très difficile pour un petit garçon de sept ans de vivre ce deuil seul!

Lors de la perte d'Ange-Albert, la peine de mes parents et l'absence subite de ce bébé alors âgé de neuf mois a sans doute perturbé mes sœurs Michelle et Thérèse. Ma sœur Michelle a tout oublié, pourtant mon frère Pierre mentionne que c'est elle qui 
était la plus affectée, car elle s'était occupée d'Ange-Albert en le berçant; elle avait 4 ans. Comment a-t-elle métabolisé cette perte? Puisque l'enfant se sent toujours coupable de la mort d'un de ses proches, elle n'a pas dû y échapper. Michel Hanus (2000, p. 39) nous dit qu'après l'âge de quatre ans, l'enfant sait que la mort est irréversible : "C'est vers l'âge de quatre ans qu'en général ils commencent à comprendre que la mort est irréversible, mais il leur faudra encore bien du temps avant de pouvoir l'accepter. » Quant à ma sœur Thérèse, qui avait deux ans à l'époque, il semble que cet événement ait encore des répercussions dans sa vie : elle m'a parlé de "choses enfouies, des choses indéfinissables » qui sont revenues à la surface quand mon frère Pierre lui a décrit la réaction qu'elle avait eue au décès d'AngeAlbert. Elle a certainement insisté pour revoir le bébé, réclamé sa présence, puisqu'à cet âge, la mort n'est pas irréversible. J'ai aussi parlé du sentiment d'abandon qui semble l'avoir marquée, ce qui serait très plausible dans les circonstances.

La mort de Gérard et celle d'Yvan, survenues alors que mes sœurs Michelle et Thérèse étaient plus âgées, ont certainement eu un impact psychologique sur elles. Ma sœur Michelle, l'aînée des filles, revivait avec effroi le moment où, à l'âge de 10 ans, elle a essuyé le sang par terre, le cœur serré en pensant perdre sa mère. Lorsqu'elle m'en a fait le récit, je voyais dans son regard la panique et l'affolement d'alors. Elle a sûrement été bouleversée et follement inquiète. Et par la suite, abattue par le tragique de cet accouchement, elle en a parlé avec ma mère et la voisine, m'a-t-elle dit. Lors de la mort de l'enfant Yvan, elle se demandait encore, soixante ans après l'événement, si on l'avait nourri avant son départ pour le baptême. Ces attitudes indiquent qu'elle a pris des responsabilités très tôt... et qu'elle a très souvent fourni un support à notre mère. Tous ces deuils l'ont certainement affectée profondément.

Ma sœur Beth dit qu'on ne lui avait pas expliqué le sens de ce qui se passait lorsque le bébé Éliane a reçu l'extrême-onction à la maison. Elle avait 5 ans à l'époque mais, à 62 ans, elle s'en souvient comme si c'était hier. Il y avait sûrement un climat d'anxiété palpable... les enfants ressentent l'affolement des adultes et l'étrangeté du moment. Ce que démontre la petite phrase répétée pendant la cérémonie par l'un des enfants présents: "Qu'est-ce qu'il fait?» Ma sœur Éliane n'est pas morte, heureusement. Mais ce récit démontre que les agonies se vivaient devant toute la famille, et que le curé a été un vrai sauveur. La description qu'en fait ma sœur Thérèse, « Il s'en venait comme un oiseau », rappelle le vol des anges venant porter secours, ou l'image de l'ange gardien qui accourt.

Je crois cependant que, comme ma mère qui a été un modèle, mes frères et sœurs ont développé leur créativité. En effet, que ce soit par la musique, le chant, le dessin, la sculpture, l'écriture, la peinture, l'ébénisterie, tous les membres de ma famille ont une activité créatrice qui est plus qu'un hobby. C'est un moyen d'expression. Je crois que grâce à cela, et au soutien de leur entourage, ils ont pu traverser ces épreuves et faire un travail de deuil, même si l'impact de ces morts d'enfants a été très fort, à tel point qu'aujourd'hui encore leurs récits témoignent des émotions vécues.

\section{INSCRIPTION DE CES MORTS D'ENFANTS DANS LA CONSTELLATION FAMILIALE}

Je dirais que toute la famille a été affectée par ces morts d'enfants, même si nous n'étions pas tous présents lorsqu'elles se sont produites. Ma mère en parlait souvent, répétant qu'elle ne savait pas comment elle avait fait pour traverser ces épreuves, mais qu'elle ne recommencerait pas. Elle nommait tous ses enfants décédés, né à telle date, mort de telle façon. Toutes et tous avaient leur place dans la famille. Je crois cependant que pour un jeune enfant, ces récits avaient quelque chose d'effrayant. En effet, avant l'âge de quatre ans, selon Hanus, la mort est contagieuse. Je cite à cet égard un extrait d'un texte que j'ai écrit à l'automne 2001 et qui met en scène un enfant d'environ trois ou quatre ans. La peur d'être contaminée par toutes ces morts (et la peur de la mort en général) me semble être un thème central de mon récit. À l'époque, je n'avais pourtant pas d'informations précises sur les réactions d'un enfant confronté à la mort et j'avais laissé mon imaginaire inventer les scènes :

Ce sont les fantômes des bébés de ma mère qui susurrent sous mon lit! La paille rêche du matelas nouvellement bourré me gratte les jambes et j'ai peur dans tout ce noir.

Je vous supplie, Jésus qui êtes au ciel, ne m'emmenez pas comme vous avez emmené les fils de ma mère [...] Tous morts et tous baptisés, oints des saintes huiles et enterrés au cimetière, une croix blanche en bois surmontant les tertres, qu'ils reposent en paix amen, in paradisum. Les anges ont déployé leurs ailes...

Recroquevillée sur mon lit de paille, je serre à deux mains mon scapulaire et ma médaille du Saint-Père. Non, non, non, je ne veux pas qu'un ange me ramasse d'un grand coup d'aile et me dépose auprès de la Vierge comme un paquet de linge sale.

Je veux rester sur la terre, même si j'ai peur de tout!

J'ai fait récemment un dessin des membres de ma famille selon leurs âges respectifs. Entre chacun des vivants, j'ai placé les enfants morts. Ils apparaissent comme des étoiles, sans corps ni visage. Cependant, pour l'enfant Ange-Albert, j'ai dessiné une forme plus cristallisée, un coquillage marin. Je crois que c'est ainsi que m'apparaissent maintenant mes frères et sœurs décédés, comme des étoiles mortes dont la lumière m'a rejointe à travers les récits de ma mère, de mon frère et de mes deux sœurs. L'enfant Ange-Albert y fait exception, car il avait déjà une relation avec les membres de ma famille, c'est pourquoi mon dessin présente une forme plus élaborée.

\section{MON DEUIL}

Je crois que le deuil de mon fils, mort à quatre mois, a été difficile parce que je n'ai pas bénéficié du soutien professionnel dont j'aurais eu besoin. Ma famille a été présente, mais avec tous ces deuils inscrits dans la mémoire familiale, cela a pu rappeler des souvenirs douloureux et influencer la communication. De plus, la perte de cet enfant est survenue alors que ma famille n'était pas dans mon entourage immédiat, dans une ville où je n'avais pas d'amis, sauf mon conjoint, qui était lui-même un étranger au pays. Il est connu aujourd'hui que la mort subite du nourrisson entraîne souvent l'éclatement du couple (CECOM, 1998). C'est ce qui m'est arrivé, car en 1970 il n'existait pas de centre de consultation concernant ce genre de deuil. Étant moi-même infirmière, censée protéger la vie, j'ai vécu ce deuil comme un échec. Nous avons donc, mon conjoint et moi, traversé ce moment avec difficultés et sans support moral adéquat.

De plus, j'ai appris quelques années plus tard qu'un médicament que j'utilisais, et que l'on employait couramment en pédiatrie contre les coliques du nourrisson, a été retiré du marché en 1985 dans le cadre d'un programme de pharmacovigilance: «Nous conseillons de ne pas administrer nos produits Phénergan aux enfants de moins de deux ans» (Fontaine, 1985).

Trop tard, monsieur le vice-président! À la lecture de ce communiqué, je suis entrée dans une immense colère et j'ai remis en question beaucoup de choses en rapport avec ma profession. Je questionne encore aujourd'hui l'aspect envahissant de la pharmacologie dans nos techniques de soins.

Cette recherche auprès de mes aînés m'a fait prendre conscience des grandes douleurs 
de ma mère et des membres de ma famille. Je crois que ma mère aurait voulu sauver tous ces enfants, malgré l'obligation à la maternité. Chacun avait sa place dans son âme. Je croyais, probablement à tort, qu'elle était peut-être soulagée d'en avoir perdu quelques-uns. Mais pourquoi ne l'aurait-elle pas été ? La machine de reproduction a ses limites. Cela n'a pu qu'augmenter son sentiment d'ambivalence, sentiment qui est normal dans tous les deuils, selon Hanus, mais qui ajoute au sentiment de culpabilité et peut mener vers un deuil compliqué. La réalité de la maternité est complexe, celle de la maternité obligée plus encore.

Je crois que cette recherche m'a permis de réparer ma relation avec ma mère et de me réparer comme sujet. $\mathrm{Si}$, comme l'écrit Janine Schasseguet-Smirgel, " la création est une impulsion profonde », le rêve que voici témoigne à mon sens d'une réparation de cette relation et donc, d'une avancée dans mon travail de deuil :

Je présente à ma mère une peinture qui représente le moulin à coudre, une surface brune. Ma mère regarde avec intérêt et me suggère ceci : "Je mettrais une rose bleue, ou plutôt six roses bleues. » Ce faisant, elle dépose les roses sur le moulin à coudre. Nous échangeons alors un regard de satisfaction et un rire complice comme si nous venions toutes les deux de résoudre un problème difficile, et que, par son geste et sa suggestion, l'œuvre se trouvait achevée!

Au réveil, je me suis souvenue que mon frère m'a dit que l'enfant Ange-Albert a été exposé sur le moulin à coudre, en face de la fenêtre. Une rose, et six roses, je crois que ce sont ses deuils et le mien. C'est ma conclusion.

\section{Note}

1. Terme emprunté à la biologie et employé en psychologie pour signifier un processus d'intégration.

\section{Bibliographie}

BLANCHET, Alain et al. (1985). L'entretien dans les sciences sociales, Paris, Dunod.

CHASSEGUET-SMIRGEL, Janine (1971). Pour une psychanalyse de l'art et de la créativité, Paris, Petite bibliothèque Payot.

Centre de communication en santé mentale (1998). Quand la vie bascule, le syndrome de mort subite du nourrisson, volet parents, vidéo, Hôpital Rivières-des-Prairies, Montréal, 85 minutes.

EHRENWALD, Jan (1981). Le lien télépathique, Paris, Robert Laffont.

FONTAINE, Roger, m.d. (1985). Lettre aux médecins, Rhone-Poulenc Pharma Inc.

HANUS, Michel (2000). «Deuils normaux, deuils compliqués, deuils pathologiques» dans La mort retrouvée, Paris, Frison-Roche. Reproduit dans les Actes du colloque Penser la mort aujourd'hui, 25 octobre 2002.

NIOBEY, Georges (dir.) (1986). Dictionnaire analogique, Paris, Larousse.

UHL, Magali (2002). « Mort et recherche. Éléments d'épistémologie complémentariste", dans P. Baudry (dir.), L'antropologie de la mort aujourd'hui. Revue de l'institut de sociologie, Université libre de Bruxelles, 1999, publié en 2002, p. 45-61. 